\title{
Plasma, tumor and tissue pharmacokinetics of Docetaxel delivered via nanoparticles of different sizes and shapes in mice bearing SKOV-3 human ovarian carcinoma xenograft
}

\author{
Kevin S. Chu, BS ${ }^{\ddagger, 1}$, Warefta Hasan, $\mathrm{PhD}^{\ddagger, 2}$, Sumit Rawal, PhD ${ }^{1}$, Mark D. Walsh, Pharm $\mathbf{D}^{1}$, \\ Elizabeth M. Enlow, $\mathrm{PhD}^{2}$, J. Christopher Luft, $\mathrm{PhD}^{2,3}$, Arlene S. Bridges, $\mathrm{PhD}^{4}$, Jennifer L. \\ Kuijer, BS ${ }^{1}$, Mary E. Napier, PhD $2,3,5$, William C. Zamboni, Pharm D, PhD $1,3,6,7,8$, and Joseph \\ M. DeSimone, PhD $^{\star}, 1,2,3,7,8,9,10,11,12$ \\ ${ }^{1}$ Department of Pharmaceutical Sciences, University of North Carolina \\ ${ }^{2}$ Department of Chemistry, University of North Carolina \\ ${ }^{3}$ Lineberger Comprehensive Cancer Center, University of North Carolina \\ ${ }^{4}$ Department of Pathology and Lab Medicine, University of North Carolina \\ ${ }^{5}$ Department of Biochemistry and Biophysics, University of North Carolina \\ ${ }^{6}$ Institute for Pharmacogenomics and Individualized Therapy, University of North Carolina \\ ${ }^{7}$ Carolina Center of Cancer Nanotechnology Excellence, University of North Carolina \\ ${ }^{8}$ Department of Pharmacology, Eshelman School of Pharmacy, University of North Carolina \\ ${ }^{9}$ Institute for Nanomedicine, University of North Carolina \\ ${ }^{10}$ Institute for Advanced Materials, University of North Carolina \\ ${ }^{11}$ Department of Chemical and Biomolecular Engineering, North Carolina State University \\ ${ }^{12}$ Sloan-Kettering Institute for Cancer Research, Memorial Sloan-Kettering Cancer Center
}

\section{Abstract}

The particle fabrication technique PRINT® was used to fabricate monodisperse size and shape specific poly(lactide-co-glycolide) particles loaded with the chemotherapeutic Docetaxel. The pharmacokinetics of two cylindrical shaped particles with diameter $=80 \mathrm{~nm}$; height $=320 \mathrm{~nm}$ (PRINT-Doc- $80 \times 320$ ) and d=200nm; h=200nm (PRINT-Doc-200×200) were compared to Docetaxel in mice bearing human ovarian carcinoma SKOV-3 flank xenografts. The Docetaxel plasma exposure was $\sim 20$-fold higher for both particles compared to docetaxel. Additionally, the volume of distribution (Vd) of Docetaxel in PRINT formulations was $~ 18$-fold (PRINTDoc-80 $\times 320$ ) and $\sim 33$-fold (PRINT-Doc- $200 \times 200$ ) lower than Docetaxel. The prolonged duration of Docetaxel in plasma when dosed with PRINT formulations subsequently lead to increased

\footnotetext{
(C) 2012 Elsevier Inc. All rights reserved.

*Corresponding Author Department of Chemistry, The University of North Carolina at Chapel Hill, Campus Box \#3290, 257 Caudill, Chapel Hill, NC 27599-3290, (919) 962-2166 phone, (919) 962-5467 fax, desimone@unc.edu.

₹Authors contributed equally to this work

Conflict of Interest Joseph DeSimone is a founder, member of the board of directors, and maintains a financial interest in Liquidia Technologies. PRINT and Fluorocur are registered trademarks of Liquidia Technologies, Inc.

Publisher's Disclaimer: This is a PDF file of an unedited manuscript that has been accepted for publication. As a service to our customers we are providing this early version of the manuscript. The manuscript will undergo copyediting, typesetting, and review of the resulting proof before it is published in its final citable form. Please note that during the production process errors may be discovered which could affect the content, and all legal disclaimers that apply to the journal pertain.
} 
tumor exposure of Docetaxel from 0-168 hours ( 53\% higher for PRINT-Doc-80×320 and $~ 76 \%$ higher for PRINT-Doc-200×200 particles). PRINT-Doc- $80 \times 320$ had lower exposures in the liver, spleen and lung compared with PRINT-Doc- $200 \times 200$. Thus, the use of particles with smaller feature size may be preferred to decrease clearance by organs of the mononuclear phagocyte system.

\section{Keywords}

soft-lithography; PLGA; nanoparticle; shape; pharmacokinetics

\section{Background}

The application of nanotechnology to oncology explores the use of macromolecular and nanoparticle carriers to enhance delivery of therapeutics and diagnostic agents. Desired outcomes of nanoparticle delivery include enhanced drug solubility, extended drug half-life, and passive targeting to solid tumors by the enhanced permeability and retention (EPR) effect [1,2] all of which may translate to improved efficacy and decreased toxicity. Two Food and Drug Administration (FDA) approved examples of nanoparticle formulations are Doxil ${ }^{\circledR}$ (pegylated liposomal doxorubicin) and Abraxane ${ }^{\circledR}$ (albumin-bound paclitaxel nanoparticle). Doxil's advantages compared to doxorubicin are increased half-life of doxorubicin in plasma and great tumor delivery and also reduced cardiotoxicity that was demonstrated in a single agent phase III study [3]. Abraxane showed benefit in response rate and progression free survival in a single agent phase III trial compared to Taxol [4]. Though there has been success in nanomedicine, the percent of injected dose of nanoparticles that reaches the tumor remains low, and thus further study of factors affecting nanoparticle tumor accumulation are warranted [5].

There are many formulation techniques for nanoparticle fabrication, including selfassembled systems such as microemulsions [6] and micelles [7], liposomes [8], emulsion/ solvent evaporation [9] and nanoprecipitation [10] based polymeric particles. Particle compositions and fabrication techniques may vary, but nanoparticles for small molecule chemotherapy delivery have similar design criteria [11-13]. Nanoparticles are typically sized larger than $10 \mathrm{~nm}$ to avoid renal clearance and extravasation to normal tissues and are smaller than $200 \mathrm{~nm}$ to reduce clearance by the liver and spleen of the mononuclear phagocyte system (MPS) [14,15]. Although general trends have been established in desired particle size for tumor accumulation and there have been some studies on the role of particle size and shape on cellular uptake of particles [16-18], few studies have explored the effect of particle shape on in vivo tumor accumulation. Geng et al demonstrated that flexible filomicelles have longer plasma circulation times and evade the MPS [19]. Chauhan et al have demonstrated that a rod shaped particle with a small diameter has better tumor penetration than spherical particles of similar hydrodynamic diameter [20].

However, to date, the interdependent effect of size and shape on chemotherapeutic tumor delivery has not been explored. In this study, we applied the PRINT ${ }^{\circledR}$ (Particle Replication In Non wetting Templates) technology, which is a soft-lithography process, to fabricate monodisperse populations of PLGA particles with high loadings of Docetaxel (Doc) [21]. PRINT is a top-down fabrication technique that produces size and shape specific particles that provides the ability to understand the role of size and shape on particle distribution in vivo [22]. Two particle shapes were used; PRINT-Doc-200×200 (PRINT-Doc-200×200) and PRINT-Doc- $80 \times 320$ cylindrical particles (PRINT-Doc-80×320). The PRINT-Doc-80×320 particle has an aspect ratio of $4: 1$. Though this particle has a longer length than the PRINTDoc- $200 \times 200$ particle, the $80 \mathrm{~nm}$ diameter may allow the particle to transport through 
smaller pores. With both particle shapes, we demonstrated improved plasma pharmacokinetics and tumor delivery compared to the approved clinical formulation of Taxotere ${ }^{\circledR}$. Additionally, differences in clearance can be seen for the two PRINT particles suggesting that shape may play a role in reducing clearance by the MPS and enhancing tumor delivery.

\section{Methods}

\section{Materials}

Poly(D,L-lactide-co-glycolide) (lactide:glycolide 85:15, $0.65 \mathrm{dL} / \mathrm{g}$ Inherent Viscosity at $30^{\circ} \mathrm{C}$ ) was purchased from Sigma-Aldrich. Chloroform and solvents (acetonitrile and water) for high performance liquid chromatography (HPLC) were purchased from Fisher Scientific. Doc was purchased from LC Laboratories. Taxotere was purchased from the University of North Carolina at Chapel Hill hospital pharmacy for research purposes. Poly(ethylene terephthalate) (PET) sheets (6" width) were purchased from KRS plastics. Fluorocur ${ }^{\circledR}$, PRINT-Doc-200×200 and PRINT-Doc- $80 \times 320$ prefabricated molds and 2,000 g/mol polyvinyl alcohol $(\mathrm{PVOH})$ coated PET sheets were provided by Liquidia Technologies.

\section{Particle Fabrication}

Doc particles were fabricated following previously published methods with modification [23]. A thin film of PLGA and Doc was deposited on a 6" $\times 12$ " sheet of PET by spreading $150 \mu \mathrm{L}$ of a $10 \mathrm{mg} / \mathrm{mL}$ PLGA and $10 \mathrm{mg} / \mathrm{mL}$ Doc chloroform solution using a 5 Mayer Rod (R.D. Specialties). The solvent was evaporated with heat. The PET sheet with the film was then placed in contact with the patterned side of a mold and passed through heated nips (ChemInstruments Hot Roll Laminator) at $130^{\circ} \mathrm{C}$ and 80 psi. The mold was split from the PET sheet as they both passed through the hot laminator. The patterned side of the mold was then placed in contact with a sheet of PET sheet coated with 2,000 g/mol PVOH. This was then passed through the hot laminator to transfer the particles from the mold to the PET sheet. The mold was then peeled from the PET sheet. The particles were removed by passing the PVOH coated PET sheet through motorized rollers and applying water to dissolve the $\mathrm{PVOH}$ to release the particles. To remove excess $\mathrm{PVOH}$, the particles were purified and then concentrated by tangential flow filtration (Spectrum Labs).

\section{Particle Characterization}

Particles were imaged by scanning electron microscopy (SEM) by pipetting a $50 \mu \mathrm{L}$ sample of particle on a glass slide. The sample was then dried and coated with $3 \mathrm{~nm}$ gold palladium alloy using a Cressington 108 auto sputter coater. Images were taken at an accelerating voltage of $2 \mathrm{kV}$ using a Hitachi model S-4700 SEM. For size and zeta potential measurement, dynamic light scattering (DLS) (Malvern Instruments Nano-ZS) was used.

\section{Drug Loading}

Doc was measured using an Agilent Technologies Series 1200 HPLC with a C18 reverse phase column (Zorbax Eclipse XDB-C18, 4.6×150mm, 5 micron). A linear gradient from $100 \%$ water to $100 \%$ acetonitrile was run over 10 minutes. Then $100 \%$ acetonitrile was run for 5 minutes. The flow rate was $1 \mathrm{~mL} / \mathrm{min}$ and detection was at $210 \mathrm{~nm}$. Particle samples were prepared by diluting the sample 1 in 10 with acetonitrile and mixing the sample to break down the particle. Standards of Doc and PLGA were prepared in acetonitrile.

\section{In Vitro Release Studies}

$100 \mu \mathrm{L}$ of particle solution $(200 \mu \mathrm{g} / \mathrm{mL}$ Doc) was placed in a mini dialysis unit with a $20 \mathrm{k}$ $\mathrm{MW}$ cutoff and dialyzed against a stirred $1 \mathrm{~L}$ bath of $1 \times \mathrm{PBS}$ at $37^{\circ} \mathrm{C}$. The bath was replaced 
periodically to maintain sink conditions. There were three dialysis units for each time point. At each time point, the particle solution in each dialysis unit was removed and centrifuged to pellet the nanoparticles. The pellet was then analyzed for the amount Doc remaining. To determine the percent Doc released over time, the amount Doc remaining was compared to the initial amount of Doc in the system.

\section{SKOV-3 human ovarian carcinoma tumor xenografts}

This study was done with an approved protocol with the University of North Carolina at Chapel Hill's Institutional Animal Care and Use Committee. All animals used were treated humanely. SKOV-3 human ovarian carcinoma cells, acquired from ATCC, were propagated in culture and harvested in log-phase growth. Female C.B.-17 SCID mice, aged 6-8 weeks and 14-18 grams in body weight, were ordered from Harlan Sprague Dawley. The mice were acclimated for 1 week prior to tumor cell injection. Cells $\left(5.0 \times 10^{6}\right.$ cells in $200 \mu \mathrm{L}$ $1 \times$ PBS) were injected subcutaneously (SC) into the right flank of each mouse. Tumor volume was calculated using the formula: tumor volume $\left(\mathrm{mm}^{3}\right)=\left(w^{2} \times I\right) / 2$, where $w=$ width and $l=$ length in $\mathrm{mm}$ of the tumor.

\section{Pharmacokinetic study}

42 days after tumor cell implantation, mice were pair matched according to tumor volume into three treatment groups. Individual tumor volumes ranged from 40 to $253 \mathrm{~mm}^{3}$ at the time of grouping. The dosage for Docetaxel administered was based upon previously published work [24]. All mice received $10 \mathrm{mg} / \mathrm{kg}$ Docetaxel via a single tail vein injection. Group $1(\mathrm{n}=17)$ received Taxotere. Group $2(\mathrm{n}=18)$ received PRINT-Doc-200 $\times 200$. Group $3(\mathrm{n}=18)$ received PRINT-Doc-80 $\times 320$. Formulations were diluted to $1 \mathrm{mg} / \mathrm{mL}$ of Docetaxel with normal saline and mice were dosed at $10 \mu \mathrm{L}$ of solution per gram of body weight.

Mice ( $\mathrm{n}=3$ per time point) were sacrificed at $0.083,1,6,24,72$, and 168 hours after dosing. Blood ( 1 mL) was collected via terminal cardiac puncture using sodium heparin as an anticoagulant under $\mathrm{CO}_{2}$ anesthesia and processed for plasma by centrifugation $(1,500 \times g$ for $5 \mathrm{~min}$ ). Plasma and tissues were placed in cryopreservation vials and preserved by snap freezing using liquid nitrogen. Tissues were stored at $-80^{\circ} \mathrm{C}$ until analysis. Samples were processed for sum total (encapsulated + released) Doc using a protein precipitation method and analyzed by LC-MS/MS.

\section{Sample Preparation and Processing}

Total tissue and tumor weight was recorded at time of collection. Whole tissue and tumors were snap frozen in liquid nitrogen and stored at $-80^{\circ} \mathrm{C}$ until homogenized. To form homogenates, the intact tissues or tumors were thawed and sectioned. The sections were weighed and diluted in a 1:3 ratio with phosphate buffered saline (PBS) solution (assumes tumor and tissue has a density of $1 \mathrm{mg} / \mathrm{ml}$ ). Finally, these mixtures were homogenized by placing zirconium oxide beads ( 15 small and 2 large) into $2 \mathrm{~mL}$ tubes at 3,000 $\times g$ using a Precellys 24 homogenizer (Bertin Technologies) twice for $15 \mathrm{sec}$ each with a $5 \mathrm{sec}$ wait between each run. The resulting homogenates were snap frozen in liquid nitrogen and stored at $-80^{\circ} \mathrm{C}$ until processed.

Sample processing for determining plasma, tissue, or tumor concentrations of Doc was similar and based on previously published methods [25]. Calibration standards, quality control samples, and dilution control samples were prepared in equivalent matrix that had demonstrated no interfering components by the addition of $10 \mu \mathrm{l}$ of a 10X solution of analyte in acidified methanol ( $0.1 \% \mathrm{v} / \mathrm{v}$ acetic acid). Dilution controls and diluted unknown samples were diluted 1:10 (10uL sample $+90 \mathrm{uL}$ appropriate matrix) prior to any processing. All 
samples, standards, and controls were processed as follows: $100 \mu \mathrm{l}$ of plasma or, tumor or tissue homogenate was pipetted into a 96-well silanized glass insert, protein-precipitated with the addition of $100 \mu \mathrm{l}$ of a 50:50 mixture of methanol:acetonitrile containing the internal standard solution (paclitaxel), vortexed for $1 \mathrm{~min}$, and centrifuged for $15 \mathrm{~min}$ at $3,000 \times \mathrm{g}$ at $4^{\circ} \mathrm{C}$. The supernatants were analyzed by liquid chromatography with detection by tandem mass spectrometry with no further manipulation needed.

\section{Liquid Chromatography Tandem Mass Spectrometry (LC-MS/MS)}

A previously described LC-MS/MS analytical method was used for the quantification of analytes [26]. A Shimadzu solvent delivery system, and an Applied Biosystems API 4000 triple quadruple mass spectrometer with an APCI ion source (Applied Biosystems) were used for these analytical studies. Separation was accomplished using a Gemini ${ }^{\circledR} \mathrm{C} 18$, $30 \times 2.0 \mathrm{~mm}$ column, with a $5 \mu \mathrm{m}$ particle size. The mass spectrometer was operated in positive ion mode using multiple reaction monitoring: DOC, $808.5 \rightarrow 527.5 \mathrm{~m} / \mathrm{z}$ and paclitaxel $854.4 \rightarrow 286.1 \mathrm{~m} / \mathrm{z}$ [26].

\section{Pharmacokinetic Analysis}

The pharmacokinetics of Taxotere, PRINT-Doc- $200 \times 200$ and PRINT-Doc- $80 \times 320$ in plasma, tumor and tissue were analyzed by noncompartmental methods using WinNonlin Professional Edition version 5.2.1 (Pharsight Corp, Cary, NC). The area under the concentration versus time curve (AUC) was calculated using the linear up/log down rule. AUC from 0 to $\mathrm{t}\left(\mathrm{AUC}_{0-\mathrm{t}}\right)$ and $\mathrm{AUC}$ from 0 to $\infty\left(\mathrm{AUC}_{0-\infty}\right)$ were calculated. Volume of distribution $\left(\mathrm{V}_{\mathrm{d}}\right)$ and clearance $(\mathrm{CL})$ were calculated using standard equations. The maximum concentration $\left(\mathrm{C}_{\max }\right)$, time of $\mathrm{C}_{\max }\left(\mathrm{T}_{\max }\right)$, last measured concentration $\left(\mathrm{C}_{\text {last }}\right)$ and time of $\mathrm{C}_{\text {last }}\left(\mathrm{T}_{\text {last }}\right)$ were determined by visual inspection of the concentration versus time curve data.

\section{Statistics}

Data was analyzed for statistical differences by one-way analysis of variance (ANOVA) followed by Bonferroni's modified t-test for multiple comparisons using GraphPad Prism (GraphPad Software, Inc., La Jolla, CA). The confidence interval was set at 95\% $(\mathrm{P}<0.05)$ to determine statistical significance.

\section{Results}

\section{Particle Fabrication}

The characteristics for the PRINT-Doc- $80 \times 320$ and PRINT-Doc- $200 \times 200$ particles are shown in Table 1 . The PRINT fabrication process makes highly monodisperse particles as visualized by the SEM images (Figure 1). The particles had slightly negative zeta potential because of the $\mathrm{PVOH}$ that remains associated with the particle following harvesting and purification. During fabrication, the particles are transferred from the mold to PVOH coated PET sheets. When the harvest sheet is dissolved with water during bead harvesting to release the particles from the sheet to solution, $\mathrm{PVOH}$ is adsorbed onto the particle surface. This slightly negative zeta potential may decrease nonspecific cellular uptake.

Particles were measured for size by DLS. Although the non-spherical particle shapes are not ideal for DLS measurement, the recorded measurements for both particle shapes were greater than $200 \mathrm{~nm}$ and the difference in hydrodynamic diameter was only $\sim 30 \mathrm{~nm}$. The similarity of hydrodynamic diameter allows for a more fair comparison of shape effects on drug pharmacokinetics. 
Additionally, as previously demonstrated [23], the Doc weight/weight percent loading (w/w $\%$ ) is much higher than what can currently be achieved with conventional bottom up formulation approaches [27-30]. The PRINT-Doc- $80 \times 320$ particles were loaded at a lower w/w\% than the PRINT-Doc- $200 \times 200$ particles $(33.5 \%$ vs $45.2 \%$ ) due to the purification process post particle fabrication. The particles were originally charged with $50 \%$ by weight of Doc. Particles were washed with sterile water and concentrated by tangential flow filtration, which allowed some Doc to leach. The PRINT-Doc- $80 \times 320$ loses a larger percentage of Doc during this purification process. This observation also matches the in vitro release profile of Doc from the particles (Figure 2). The PRINT-Doc-80×320 particles have a greater burst release than the PRINT-Doc- $200 \times 200$ particles. $~ 60 \%$ of the Doc is released in vitro for PRINT-Doc- $80 \times 320$ particles by 3 hours compared to $~ 38 \%$ for the PRINT-Doc- $200 \times 200$ particles. Additionally, at 24 hours, nearly $100 \%$ of drug is released from the PRINT-Doc- $80 \times 320$ particles mean while the PRINT-Doc- $200 \times 200$ particles still hold $\sim 27 \%$ of its cargo. The difference in release may be dictated by the particle geometry. Per unit volume, a PRINT-Doc- $80 \times 320$ particle has more surface area compared to a PRINT-Doc- $200 \times 200$ particle. This calculation assumes the PRINT-Doc- $80 \times 320$ particle is a cylinder that is $80 \mathrm{~nm}$ in diameter and has a length of $320 \mathrm{~nm}$ and assumes the PRINTDoc- $200 \times 200$ particle is a cylinder that is $200 \mathrm{~nm}$ in diameter and has a length of $200 \mathrm{~nm}$. Increased surface area per unit volume leads to faster drug release.

\section{Pharmacokinetics of PRINT particles and Taxotere}

Sum total (encapsulated and released) docetaxel was measured for each organ. The concentration versus time profiles of Taxotere, PRINT-Doc-200×200 and PRINTDoc- $80 \times 320$ in plasma, tumor, spleen, liver and lungs are presented in Figure 3 . The pharmacokinetic parameters of Taxotere, PRINT-Doc-200×200 and PRINT-Doc-80×320 in plasma, tumor, spleen, liver and lungs are presented in Table 2. The PRINT particles had $\sim 20$-fold higher plasma exposure as measured by AUC compared to Taxotere. The PRINTDoc- $80 \times 320$ and PRINT-Doc-200×200 particles had $\sim 5$-fold and $\sim 7$-fold higher maximal plasma Doc concentration than Taxotere, respectively. The difference in $\mathrm{C}_{\max }$ was statistically significant higher for both PRINT particles compared to Taxotere $(\mathrm{P}<0.05)$. Additionally, the volume of distribution was much lower for the PRINT particles compared to Taxotere. The $\mathrm{V}_{\mathrm{d}}$ for PRINT-Doc- $80 \times 320$ and PRINT-Doc-200 $\times 200$ was $\sim 18$-fold and $\sim 33$-fold, respectively, less than that of Taxotere. Encapsulation of Doc into PRINT particles also decreased the clearance by $\sim 24$-fold compared to Taxotere.

The PRINT-Doc- $80 \times 320$ and PRINT-Doc-200×200 particles had a 53\% and 76\% increase in total tumor Doc exposure compared to Taxotere from 0 to $168 \mathrm{~h}$. Interestingly, looking at the tumor concentration versus time curve for 0 to 24 hours, the PRINT-Doc-80×320 particles gave a higher Doc exposure than the PRINT-Doc-200×200 particles, despite PRINT-Doc- $200 \times 200$ particles having higher exposure from $0-168$ hours. The tumor AUC $_{0-24 \mathrm{~h}}$ for PRINT-Doc- $80 \times 320$ was $20 \%$ higher than PRINT-Doc-200 $\times 200$, but for $\mathrm{AUC}_{0-168}$, the value for PRINT-Doc- $200 \times 200$ particles was $\sim 15.5 \%$ higher than that of PRINT-Doc- $80 \times 320$. Also, the maximal tumor Doc concentration was at 1 hour for the PRINT-Doc-200×200 particles as opposed to 6 hours for the PRINT-Doc- $80 \times 320$ particles. Additionally, the docetaxel concentration at 24 hours was higher for the $80 \times 320 \mathrm{~nm}$ particles compared to the $200 \times 200 \mathrm{~nm}$ particles $(387 \mathrm{ng} / \mathrm{mL}$ versus $27 \mathrm{ng} / \mathrm{mL}$ ). This indicates that the PRINT-Doc- $80 \times 320$ may have steady accumulation at the site of the tumor. The plasma AUC for 0-24 hours and 0-168 hours of the PRINT-Doc- $80 \times 320$ and PRINT-Doc-200×200 particles are similar. Thus, for the same plasma exposure from 0-24 hours, it appears that the PRINT-Doc- $80 \times 320$ is more efficient at delivering Doc to the tumor than the PRINTDoc- $200 \times 200$ particle. However, from 0-168 hours, for similar plasma exposure, the PRINT-Doc-200×200 gave higher Doc exposure in the tumor. The $\mathrm{C}_{\text {last }}$ of the PRINT- 
Doc- $200 \times 200$ particles was higher in the tumor than the PRINT-Doc- $80 \times 320$ particles. Tweaking the PRINT-Doc- $80 \times 320$ particle to decrease drug release rate may increase Doc exposure at the tumor at longer time points.

Both particles had higher Doc exposure in the spleen and liver compared to Taxotere as expected for a nanoparticle formulation [5,12-13]. However, the PRINT-Doc-200×200 particles had $\sim 4.8$-fold higher Doc exposure in the spleen compared to the PRINTDoc- $80 \times 320$ particles. The maximal spleen concentration was also higher for the PRINTDoc-200×200 particles compared to PRINT-Doc- $80 \times 320(18,038 \mathrm{ng} / \mathrm{mL}$ vs $32,333 \mathrm{ng} / \mathrm{mL}$, statistically significant). The spleen docetaxel concentration for the $200 \times 200 \mathrm{~nm}$ particles was also higher than the $80 \times 320 \mathrm{~nm}$ particles at all times points after 5 minutes. Despite the longer $320 \mathrm{~nm}$ dimension, the higher aspect ratio PRINT-Doc- $80 \times 320$ particle had less Doc exposure in the spleen compared to the PRINT-Doc- $200 \times 200$ particles.

The liver Doc exposure for the PRINT-Doc-200×200 particle was 1.4-fold higher than PRINT-Doc-80×320 particles for $\mathrm{AUC}_{0-24 \mathrm{~h}}$. However, the maximal concentrations were not significantly different. The lung Doc exposure for the PRINT-Doc-200×200 particle was also 1.4-fold higher than PRINT-Doc- $80 \times 320$ particles. The PRINT-Doc- $200 \times 200$ particles also gave a higher maximal Doc concentration in the lungs compared to the PRINTDoc- $80 \times 320$ particles, which was statistically significant. The PRINT-Doc-200×200 particles, possible due to its larger diameter, gets cleared more in organs such as the lung, liver and spleen compared to the PRINT-Doc- $80 \times 320$ particles.

\section{Discussion}

Monodisperse size and shape specific PLGA Doc nanoparticles were fabricated by the PRINT process. These particles had very high loadings of Doc relative to other nanoparticles of Docetaxel [27-30]. Although the role of drug loading on drug pharmacokinetics and efficacy has not yet been established, higher drug loaded particles allows for less of the non-active excipients to be injected. The formulations Taxotere and Taxol may cause adverse reactions related to the surfactants used (polyoxyethylated castor oil and tween 80) [31,32]. Thus, injecting less non active excipient relative to active drug may increase tolerability of the formulation, especially as related to infusion related reactions $[4,33]$.

The PRINT particles resulted in much higher plasma exposures of Doc compared to Taxotere. Accordingly, the volume of distribution and clearance of the PRINT particles was reduced related to Taxotere. Encapsulation of Doc into PRINT nanoparticles keeps the Doc more confined to the plasma compartment to allow for longer circulation and subsequently increased tumor accumulation. Additionally, reduced distribution to normal tissues may enhance the tolerability of the PRINT formulation compared to Taxotere. Furthermore, the two particles had similar plasma Doc exposure, but from 0-24 hours, the PRINT-

Doc- $80 \times 320$ particle had higher tumor Doc exposure compared with PRINT-Doc-200×200. Thus, though different particles may have longer circulation times and higher plasma drug exposure, the shape of the particle may play a role in the efficiency of delivery to the tumor. Because minimal amount of drug compared to total dose administered reaches the tumor, incremental changes to improve tumor delivery and transport may prove to be worthwhile.

Shape selection may also aid in reducing nanoparticle clearance from MPS related organs such as the spleen and liver. Despite its longer $320 \mathrm{~nm}$ dimension, the PRINT-Doc-80×320 particle had reduced exposure in the spleen, liver and lung than the PRINT-Doc-200×200 particle. Thus, the smallest dimension of the particle may be the determining factor of particle clearance. On a similar note, Chauhan et al found that $\mathrm{d}=14 \mathrm{~nm} ; \mathrm{h}=55 \mathrm{~nm}$ rods 
had the same tumor transport as $13 \mathrm{~nm}$ PEG-coated CdSe/CdS quantum dots, and concluded that the smallest dimension may be the determining factor in tumor transport [20]. Thus, future particle design may be dictated by choosing smaller particle diameters for better tumor delivery and MPS evasion.

However, though particles with smaller diameter may be preferred for enhanced passive targeting applications, smaller particles will typically have increased drug release rates due to increased surface to volume ratio. This likely explains the higher docetaxel levels for PRINT-Doc- $80 \times 320$ particles in the tumor from 0-24 hours, but not from 0-168 hours compared to the PRINT-Doc-200×200 particles. Decreasing release rate may also be preferred to keep docetaxel within the particle while the majority of particles are still circulating within the first 24 hours after administration. Studies are currently on going to determine the effect of drug release rate on pharmacokinetics and biodistribution in particles of the same size that have varied release rate.

Fabrication of particles by the PRINT produces monodisperse particles of specific size and shape that allows for the study of the effects of size and shape on drug distribution. In this study, the effect of size and shape on Doc pharmacokinetics was studied using a higher aspect ratio shaped PRINT-Doc- $80 \times 320$ particle and a PRINT-Doc-200×200 particle. Both particles were compared to the clinical comparator for Doc, Taxotere. The PRINT-

Doc-80×320 and PRINT-Doc-200×200 particles both resulted in much higher Doc plasma levels and also greatly decreased distribution volume and clearance. The increase in Doc plasma exposure due to Docparticle encapsulation led to increased tumor Doc exposure for both particles compared to Taxotere. The PRINT-Doc- $80 \times 320$ particle had higher tumor Doc accumulation from 0-24 hours and also higher Doc plasma levels than the PRINTDoc- $200 \times 200$ particles at 24 hours. Additionally, the PRINT-Doc- $80 \times 320$ particle had significantly less Doc exposure in the spleen as well as the liver and lungs. Though both particles had improved pharmacokinetics over Taxotere, the PRINT-Doc- $80 \times 320$ particle may be preferred for long circulation due to its smaller diameter to penetrate pores, which results in better evasion of the MPS and higher tumor accumulation.

\section{Acknowledgments}

Sources of funding This work was supported by the Carolina Center for Nanotechnology Excellence (U54CA151652 and U54-CA119343), University Cancer Research Fund, and Liquidia Technologies, Inc.

\section{References}

1. Matsumura Y, Maeda H. A new concept for macromolecular therapeutics in cancer chemotherapy: mechanism of tumoritropic accumulation of proteins and the antitumor agent smancs. Cancer Res. 1986; 46:6387-92. [PubMed: 2946403]

2. Maeda H, Wu J, Sawa T, Matsumura Y, Hori K. Tumor vascular permeability and the EPR effect in macromolecular therapeutics: a review. J Control Release. 2000; 65:271-84. [PubMed: 10699287]

3. O'Brien MER. Reduced cardiotoxicity and comparable efficacy in a phase III trial of pegylated liposomal doxorubicin $\mathrm{HCl}$ (CAELYXTM/Doxil") versus conventional doxorubicin for first-line treatment of metastatic breast cancer. Ann Oncol. 2004; 15:440-449. [PubMed: 14998846]

4. Gradishar WJ, Tjulandin S, Davidson N, Shaw H, Desai N, Bhar P, et al. Phase III trial of nanoparticle albumin-bound paclitaxel compared with polyethylated castor oil-based paclitaxel in women with breast cancer. J Clin Oncol. 2005; 23:7794-803. [PubMed: 16172456]

5. Chrastina A, Massey KA, Schnitzer JE. Overcoming in vivo barriers to targeted nanodelivery. Wiley Interdiscip Rev Nanomed Nanobiotechnol. 2011; 3:421-37. [PubMed: 21538941]

6. Dong X, Mattingly CA, Tseng MT, Cho MJ, Liu Y, Adams VR, et al. Doxorubicin and paclitaxelloaded lipid-based nanoparticles overcome multidrug resistance by inhibiting P-glycoprotein and depleting ATP. Cancer Res. 2009; 69:3918-26. [PubMed: 19383919] 
7. Kim SC, Kim DW, Shim YH, Bang JS, Oh HS, Wan Kim S, et al. In vivo evaluation of polymeric micellar paclitaxel formulation: toxicity and efficacy. J Control Release. 2001; 72:191-202. [PubMed: 11389998]

8. Needham D, Anyarambhatla G, Kong G, Dewhirst MW. A new temperature-sensitive liposome for use with mild hyperthermia: characterization and testing in a human tumor xenograft model. Cancer Res. 2000; 60:1197-201. [PubMed: 10728674]

9. Doiron AL, Chu K, Ali A, Brannon-Peppas L. Preparation and initial characterization of biodegradable particles containing gadolinium-DTPA contrast agent for enhanced MRI. Proc Natl Acad Sci USA. 2008; 105:17232-7. [PubMed: 18796605]

10. Gu F, Zhang L, Teply BA, Mann N, Wang A, Radovic-Moreno AF, et al. Precise engineering of targeted nanoparticles by using self-assembled biointegrated block copolymers. Proc Natl Acad Sci USA. 2008; 105:2586-91. [PubMed: 18272481]

11. Farokhzad O, Langer RS. Nanoparticle Delivery of Cancer Drugs. Annu Rev Med. 2011; 63:185198. [PubMed: 21888516]

12. Petros RA, DeSimone JM. Strategies in the design of nanoparticles for therapeutic applications. Nat Rev Drug Discov. 2010; 9:615-27. [PubMed: 20616808]

13. Yoo JW, Chambers E, Mitragotri S. Factors that control the circulation time of nanoparticles in blood: challenges, solutions and future prospects. Curr Pharm Des. 2010; 16:2298-307. [PubMed: 20618151]

14. Choi HS, Liu W, Misra P, Tanaka E, Zimmer JP, Itty Ipe B, et al. Renal clearance of quantum dots. Nat Biotechnol. 2007; 25:1165-70. [PubMed: 17891134]

15. Chen LT, Weiss L. The role of the sinus wall in the passage of erythrocytes through the spleen. Blood. 1973; 41:529-37. [PubMed: 4688868]

16. Champion JA, Mitragotri S. Shape induced inhibition of phagocytosis of polymer particles. Pharm Res. 2009; 26:244-9. [PubMed: 18548338]

17. Gratton SE, Ropp PA, Pohlhaus PD, Luft JC, Madden VJ, Napier ME, et al. The effect of particle design on cellular internalization pathways. Proc Natl Acad Sci USA. 2008; 105:11613-8. [PubMed: 18697944]

18. Sharma G, Valenta DT, Altman Y, Harvey S, Xie H, Mitragotri S, et al. Polymer particle shape independently influences binding and internalization by macrophages. J Control Release. 2010; 147:408-12. [PubMed: 20691741]

19. Geng Y, Dalhaimer P, Cai S, Tsai R, Tewari M, Minko T, et al. Shape effects of filaments versus spherical particles in flow and drug delivery. Nat Nanotechnol. 2007; 2:249-55. [PubMed: 18654271]

20. Chauhan VP, Popovi Z, Chen O, Cui J, Fukumura D, Bawendi MG, et al. Fluorescent nanorods and nanospheres for real-time in vivo probing of nanoparticle shape-dependent tumor penetration. Angew Chem Int Ed Engl. 2011; 50:11417-20. [PubMed: 22113800]

21. Rolland JP, Maynor BW, Euliss LE, Exner AE, Denison GM, DeSimone JM. Direct fabrication and harvesting of monodisperse, shape-specific nanobiomaterials. J Am Chem Soc. 2005; 127:10096-100. [PubMed: 16011375]

22. Jeong W, Napier ME, DeSimone JM. Challenging nature's monopoly on the creation of welldefined nanoparticles. Nanomedicine (Lond). 2010; 5:633-9. [PubMed: 20528457]

23. Enlow EM, Luft JC, Napier ME, DeSimone JM. Potent engineered PLGA nanoparticles by virtue of exceptionally high chemotherapeutic loadings. Nano Lett. 2011; 11:808-13. [PubMed: 21265552]

24. Zamboni WC, Strychor S, Joseph E, Parise RA, Egorin MJ, Eiseman JL. Tumor, tissue, and plasma pharmacokinetic studies and antitumor response studies of Docetaxel in combination with 9-nitrocamptothecin in mice bearing SKOV-3 human ovarian xenografts. Cancer Chemother Pharmacol. 2008; 62:417-26. [PubMed: 17957368]

25. Hou W, Watters JW, McLeod HL. Simple and rapid Docetaxel assay in plasma by protein precipitation and high-performance liquid chromatography-tandem mass spectrometry. $\mathrm{J}$ Chromatogr B Analyt Technol Biomed Life Sci. 2004; 804:263-7. 
26. Zamboni WC, Combest AJ, DeLoia JA, Edwards RP, Bridges AS, Zamboni BA, et al. Pharmacologic and phenotypic study of Docetaxel in patients with ovarian or primary peritoneal cancer. Cancer Chemother Pharmacol. 2011; 68:1255-62. [PubMed: 21437702]

27. Cheng J, Teply BA, Sherifi I, Sung J, Luther G, Gu FX, et al. Formulation of functionalized PLGA-PEG nanoparticles for in vivo targeted drug delivery. Biomaterials. 2007; 28:869-76. [PubMed: 17055572]

28. Murugesan S, Ganesan S, Averineni RK, Nahar M, Mishra P, Jain NK. PEGylated Poly(Lactideco-Glycolide) (PLGA) Nanoparticulate Delivery of Docetaxel: Synthesis of Diblock Copolymers, Optimization of Preparation Variables on Formulation Characteristics and In Vitro Release Studies. J Biomed Nanotechnol. 2007; 3:52-60.

29. Musumeci T, Ventura CA, Giannone I, Ruozi B, Montenegro L, Pignatello R, et al. PLA/PLGA nanoparticles for sustained release of Docetaxel. Int J Pharm. 2006; 325:172-9. [PubMed: 16887303]

30. Senthilkumar M, Mishra P, Jain NK. Long circulating PEGylated poly(D,L-lactide-co-glycolide) nanoparticulate delivery of Docetaxel to solid tumors. J Drug Target. 2008; 16:424-35. [PubMed: 18569287]

31. Dye D, Watkins J. Suspected anaphylactic reaction to Cremophor EL. Br Med J. 1980; 280:1353. [PubMed: 7388538]

32. Kadoyama K, Kuwahara A, Yamamori M, Brown JB, Sakaeda T, Okuno Y. Hypersensitivity reactions to anticancer agents: data mining of the public version of the FDA adverse event reporting system, AERS. J Exp Clin Cancer Res. 2011; 30:93. [PubMed: 21970649]

33. ten Tije AJ, Verweij J, Loss WJ, Sparreboom A. Pharmacological effects of formulation vehicles: implications for cancer therapy. Clin Pharmacokinet. 2003; 42:665-85. [PubMed: 12844327] 


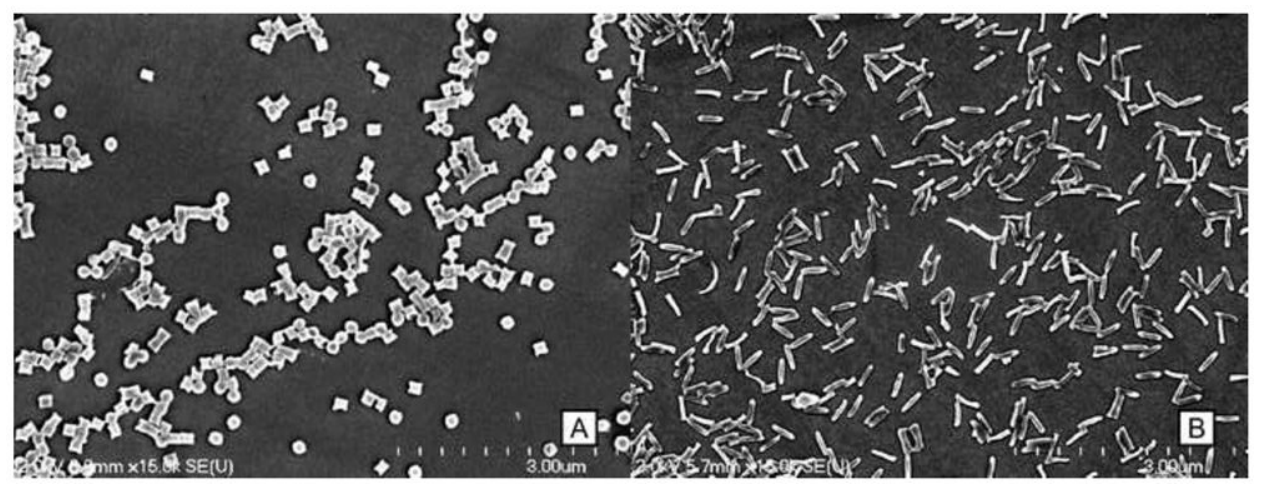

Figure 1.

Scanning electron microscopy image of (A) PRINT-Doc-200×200 particles and (B) PRINTDoc- $80 \times 320$ particles 


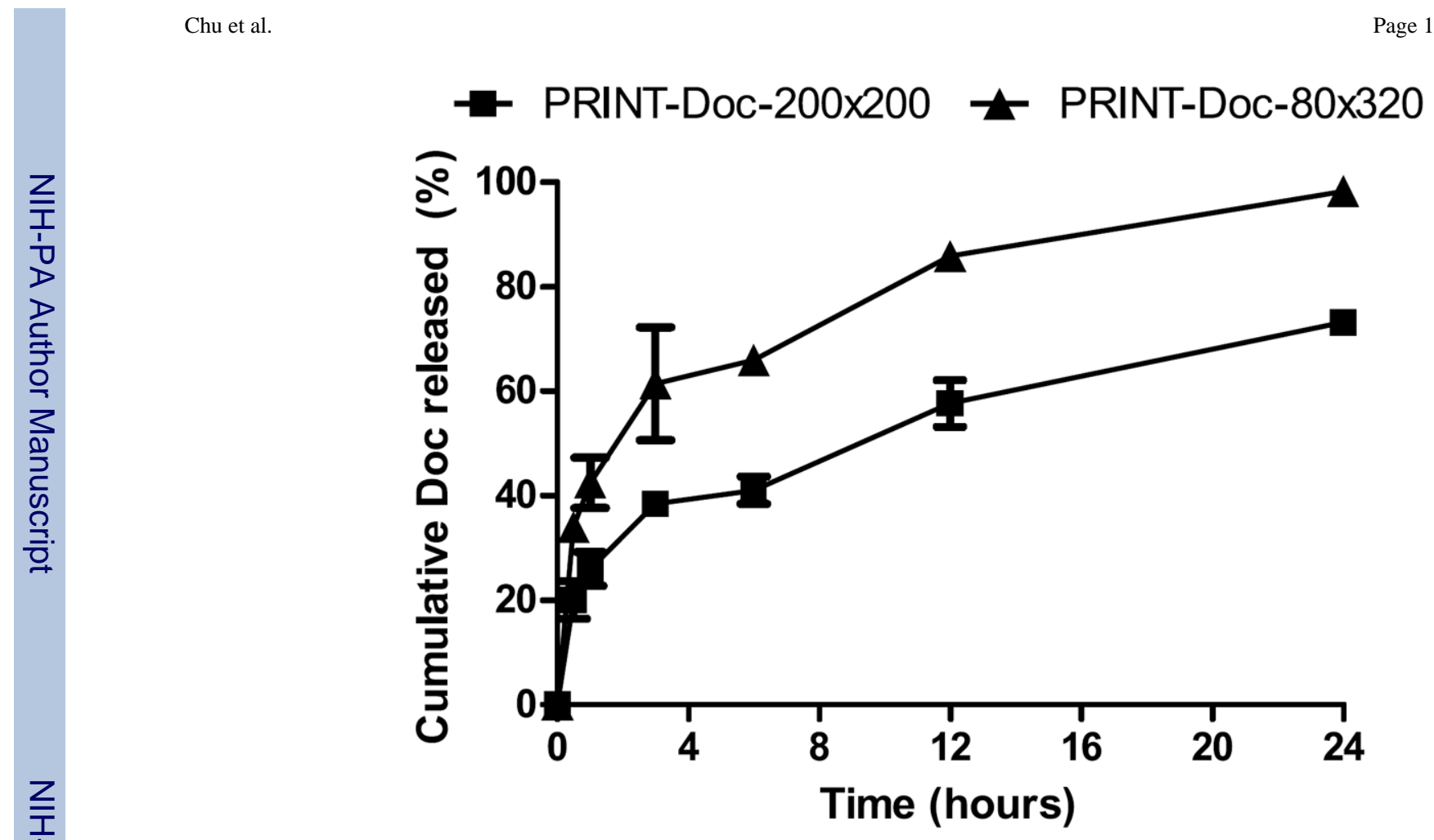

Figure 2.

Percent Docetaxel released from PRINT-Doc- $80 \times 320$ and PRINT-Doc-200×200 particles when incubated at $37^{\circ} \mathrm{C}$ in $1 \times$ PBS. for PRINT-Doc- $200 \times 200$ particles and $\boldsymbol{\Delta}$ for PRINTDoc- $80 \times 320$ particles. $\mathrm{N}=3$ measurements per time point. 


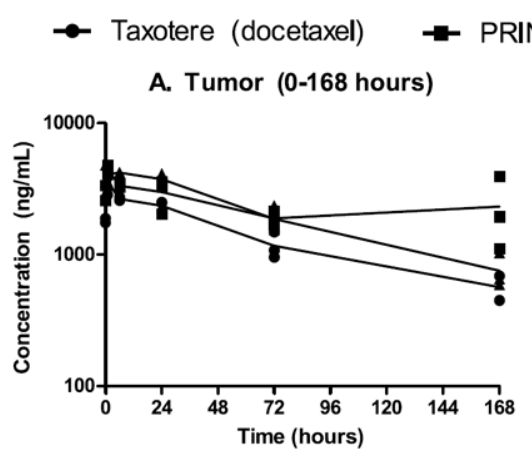

PRINT-Doc-200×200

PRINT-Doc-80×320
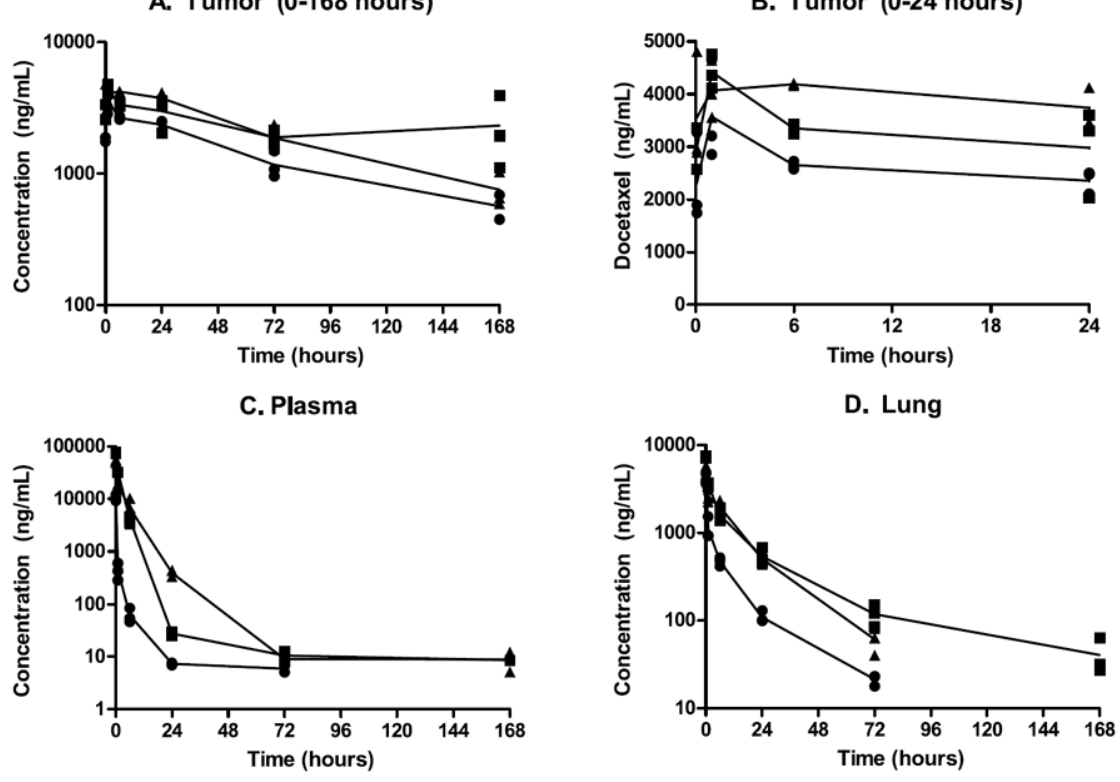

E. Spleen

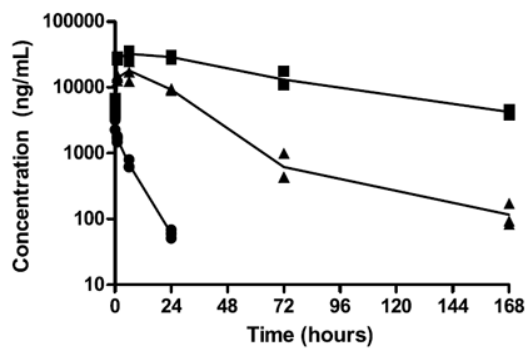

F. Liver

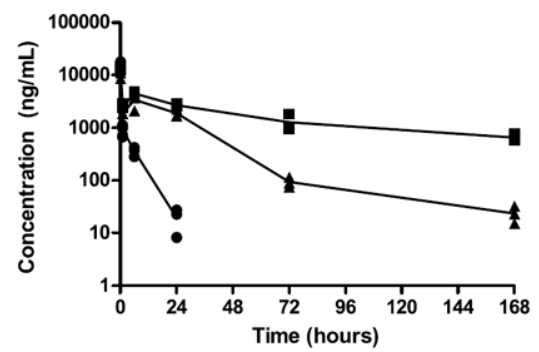

Figure 3.

Docetaxel concentration versus time curve for (A) Tumor (0-168 hours), (B) Tumor (0-24 hours), (C) Plasma, (D) Lung, (E) Spleen and (F) Liver. Docetaxel concentration values for each mouse are represented by for Taxotere, $\mathbf{a}$ for PRINT-Doc-200×200 particles and $\boldsymbol{\Delta}$ for PRINT-Doc- $80 \times 320$ particles. The lines are connected by the mean value for each time point. 
Table 1

Characterization of particles used in the pharmacokinetics study.

\begin{tabular}{|lcccc|}
\hline Particle & Doc w/w\% & Size $(\mathbf{n m})$ & PDI & Zeta Potential $(\mathbf{m V})$ \\
\hline PRINT-Doc- $80 \times 320$ & 33.5 & $227 \pm 10$ & $0.18 \pm 0.03$ & $-3.2 \pm 0.5$ \\
PRINT-Doc-200×200 & 45.2 & $263 \pm 1.8$ & $0.09 \pm 0.01$ & $-3.4 \pm 0.5$ \\
\hline
\end{tabular}




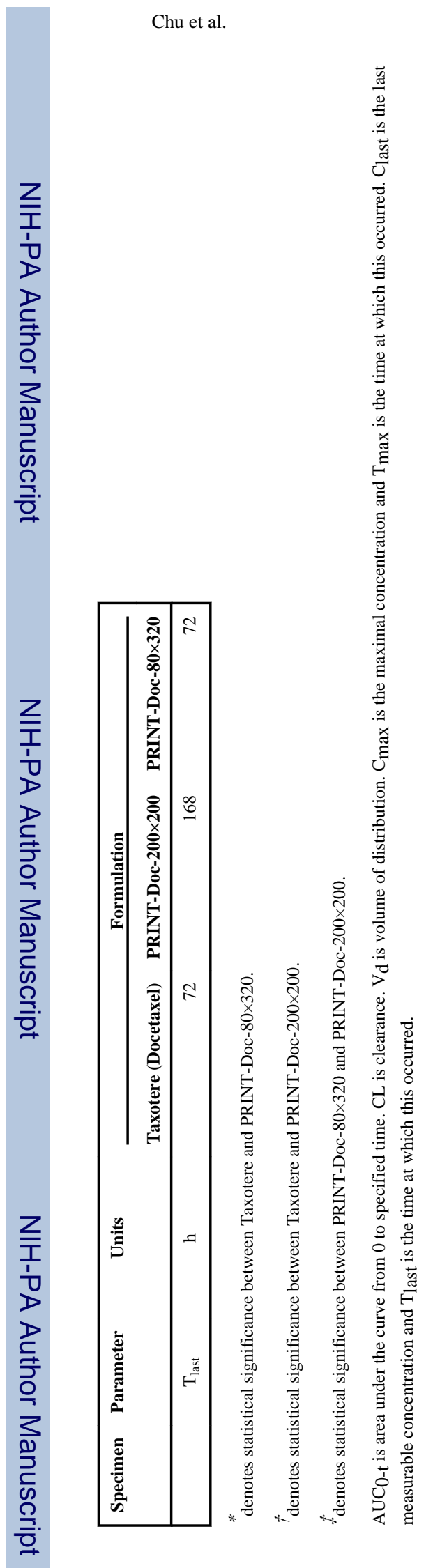

Page 16 